\title{
Synchronization of Timing And Motion Among Performing Musicians
}

\section{Werner Goebl and Caroline Palmer McGill University, Montreal, Canada}

WE INVESTIGATED INFLUENCES OF AUDITORY FEEDBACK, musical role, and note ratio on synchronization in ensemble performance. Pianists performed duets on a piano keyboard; the pianist playing the upper part was designated the leader and the other pianist was the follower. They received full auditory feedback, one-way feedback (leaders heard themselves while followers heard both parts), or self-feedback only. The upper part contained more, fewer, or equal numbers of notes relative to the lower part. Temporal asynchronies increased as auditory feedback decreased: The pianist playing more notes preceded the other pianist, and this tendency increased with reduced feedback. Interonset timing suggested bidirectional adjustments during full feedback despite the leader/follower instruction, and unidirectional adjustment only during reduced feedback. Motion analyses indicated that leaders raised fingers higher and pianists' head movements became more synchronized as auditory feedback was reduced. These findings suggest that visual cues became more important when auditory information was absent.

Received July 14, 2008, accepted February 13, 2009.

Key words: synchronization, timing, motion, ensemble performance, auditory feedback

$\mathrm{M}$ OST FORMS OF ENSEMBLE PERFORMANCE require musicians to coordinate their actions with one another in order to realize a common musical goal. These requirements go beyond the cognitive and motor demands imposed by solo music performance (e.g., Altenmüller, Wiesendanger, \& Kesselring, 2006; Palmer, 1997) in that the expressive parameters - particularly the timing of tone onsets - of each musical part or instrument have to be synchronized with those of the other performers. Performers' familiarity with the specific musical piece and the performance style can assist their ability to anticipate the timing of events. Certain musical ensemble configurations or particular musical contexts specifically require performers to adjust their timing in response to that of fellow performers; for example, the performer playing an accompaniment often has to adapt to the performer playing the melodic line. In the absence of a conductor, ensemble performers must pay attention to (by listening and watching) each others' performance in order to adjust their performance accordingly. The present study investigated the synchronization of sound and movement among performers in piano duets as a function of auditory feedback, the musical roles of leader and follower, and the relative number of events in each musical part.

Auditory feedback in expressive music performance is an important component of the action-perception link that musicians establish through practice on their instrument. Removing auditory feedback does not immediately alter solo performance of simple welllearned pieces (Finney, 1997), but it deteriorates the learning of novel music (Finney \& Palmer, 2003). Delaying auditory feedback disrupts the timing of performance considerably (Pfordresher \& Palmer, 2002). Synchronization relies generally on multimodal information. In finger tapping with a metronome, synchronization is achieved by using information from other modalities such as proprioception when auditory feedback from the taps is not made available (Aschersleben \& Prinz, 1995). In ensemble performance, auditory feedback from the other ensemble members is likely to be important for synchronization. In this study, we investigated ensemble synchronization under conditions in which performers have full or no auditory feedback of the other performer in order to examine potential consequences on other communication modalities, in particular visual information of the other performer.

Visual communication (through gestures, eye contact, body sway, etc.) has been shown to be important in music performance. Large body movements during performance ("ancillary gestures," Delalande, 1988) can influence viewers' knowledge about performers' expressive intent (Davidson, 1993) and influence perceivers' aesthetic experience (Vines, Krumhansl, Wanderley, \& Levitin, 2006). For example, head movements differ

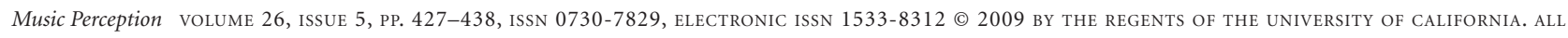
RIGHTS RESERVED. PLEASE DIRECT ALL REQUESTS FOR PERMISSION TO PHOTOCOPY OR REPRODUCE ARTICLE CONTENT THROUGH THE UNIVERSITY OF CALIFORNIA PRESS'S RIGHTS AND PERMISSIONS WEBSITE, HTTP://WWW.UCPRESSJOURNALS.COM/REPRINTINFO.ASP. DOI:10.1525/MP.2009.26.5.427 
with emotional expression; pianists' head movement velocities differed systematically with expressive intent (Castellano, Mortillaro, Camurri, Volpe, \& Scherer, 2008), and marimba players' head movements influenced listeners' ratings of expressive intent (Dahl \& Friberg, 2007). Visual communication is important for ensemble performance as well; for example, a study of two pianists who rehearsed and performed piano duet compositions (Williamon \& Davidson, 2002) revealed increasing amounts of communicative head bends and eye contact over rehearsal time.

Synchronization in ensemble performance also may be influenced by specific musical roles of the performers that determine who is the leader or follower. Strategies may range from obedient following (tracking or "hunting," Goodman, 2002) to fully democratic "cooperation" in which both musicians react to one another equally. Musical roles ("soloist" versus "accompanist," Moore, 1979) entail social as well as musical relationships (e.g., Maduell \& Wing, 2007; Murnighan \& Conlon, 1991). Davidson and Good (2002) used an observation and interview paradigm to investigate the specific musical consequences of the complex interpersonal dynamics in a string quartet rehearsing and performing. Keller (2008) studied how individual performers' attentional and memory processes influence musical ensemble performance. He derived measures of individual "anticipatory auditory imagery," "prioritized integrative attention," and "adaptive timing" and showed that they all correlated positively with body sway synchronicity in piano duets (a measure of ensemble cohesion). At least one member of the duet (but not both) needed high scores in those ensemble skill measures to obtain good overall ensemble cohesion, suggesting that the participants may have adopted musical roles as leader and follower. In the present study, we specifically addressed the consequences of being the leader or follower in a piano duet.

Although there are few studies on musicians' ensemble timing (Rasch, 1979; Shaffer, 1984), there is a large corpus of tapping studies in which individuals synchronize their taps with an auditory sequence (see Repp, 2005, for a review). Data from these studies have been analyzed using linear models (e.g., Vorberg \& Wing, 1996) or nonlinear dynamical systems models (e.g., Large \& Jones, 1999; Large \& Kolen, 1994). These models generally assume two internal processes of adaptive timing: period correction and phase correction (e.g., Mates, 1994). Period correction refers to the adjustment of an internal timekeeper; it normally occurs only when the tempo of the acoustic referent changes (Repp, 2005). Phase correction, on the other hand, is responsible for adjusting the times of successive taps whilst leaving the period of the timekeeper unchanged. A simple first-order linear phase correction model by Vorberg and Schulze (2002, p. 62) predicts larger intertap interval variability as the degree of phase correction increases. Thus, the timing variability might be indicative of the degree of timing adaptation in duet performances.

Piano duet performance usually involves more complex interaction modes than the 1:1 tapping of many tapping studies. The score often requires one musician to produce more tones in succession than the other, depending on the rhythmic complexity of the piece (e.g., a pianist may have to adjust $1 / 16$ note accompaniment passages to a few melody notes played by the other pianist or vice versa). Tapping along with a subdivided metronome beat shows less variability than tapping with a simple beat ("subdivision benefit," Repp, 2003). ${ }^{1}$ Another study showed that subdividing a beat (by producing additional movements) reduces the mean synchronization error (Wohlschläger \& Koch, 2000). In contrast, Loehr and Palmer (2009) found reduced mean synchronization error when pianists' beats were subdivided by self-produced tones versus computer-produced tones. We were interested in whether such a subdivision effect also can be found in piano duet performance. In the current study, we manipulated the relative density of musical tones in each part, resulting in different "note ratios" between parts, to address this question.

Ensemble pianists not only try to align their produced sounds, but may also synchronize their finger movements and other gestures (a tendency that might be increased in a reduced feedback condition in which they cannot hear each other). Finger motion may be influenced by factors documented in solo performanceincluding biomechanical and cognitive factors-that have not yet been addressed in the context of piano duet performance. One tapping study showed that pianists' finger trajectories were constrained more by biomechanical factors (the coupling between adjacent fingers) than by cognitive factors (such as those imposed by grouping and meter, Loehr \& Palmer, 2007). Other studies indicated that the kinematic properties of finger movements in piano performance change with performance rate: Pianists raised their

\footnotetext{
${ }^{1}$ This benefit only extends down to subdivision intervals of about $200 \mathrm{~ms}$ (Repp, 2003). This threshold is just below the durations of the eighth notes in the present study.
} 
fingers higher above the keyboard as the tempo became faster (Palmer \& Dalla Bella, 2004) and finger movements contained more kinematic landmarks that originated from the impact of the finger on the key surface as the tempo became faster (Goebl \& Palmer, 2008). These finger-key (FK) landmarks yielded tactile information that influenced the timing accuracy of subsequent events. FK landmarks are also indicators of a pianist's touch and result from different movement trajectories during a keystroke. It is possible that such kinematic cues play a role in duet synchronization under reduced auditory feedback conditions.

The purpose of the present study was to examine the synchronization of sound and body movements in piano duets while auditory feedback, instructed musical role, and note ratio between the two musical parts were manipulated. The auditory feedback that the two pianists received from each other was either full (natural), one-way (the leaders heard only themselves while the followers heard both parts), or self-feedback only. We were interested in whether the pianists could still play in synchrony when they could not hear each other. Furthermore, we asked the participants to adopt musical roles as leaders and followers. We examined whether they could realize these roles, such that the leader really led the music and did not react to the timing of the follower. The musical roles as leaders and followers were enforced in the one-way feedback condition (in which followers heard both parts, while leaders heard only themselves) to allow the comparison of synchronization accuracy with conditions of natural auditory feedback. And finally, we varied the note ratio between the performers' musical parts (1:1, $2: 1,1: 2)$ to ask: Is it easier to follow when more tone onsets are available than when there are fewer onsets? The effects of these variables on synchronization accuracy were measured in terms of the timing of individual keystrokes, properties of the finger kinematics, and aspects of upper body movements of the two pianists performing together.

\section{Method \\ Participants}

Sixteen highly trained pianists from the Montreal area participated in the study. They were 21.9 years of age on average (18 to 32 years) and had received 14.4 years of piano instruction on average (10 to 27 years). All participants gave informed consent and were paid a nominal fee for their participation. They all reported normal hearing; all but one reported being right-handed.

\section{Stimulus Materials}

Three two-part pieces were created that were easy to perform and were also musically appropriate for the interpretation of one part as the "leader." They are shown in Figure 1b. The pieces differed in ratio of notes between the parts: The first piece had quarter notes in both parts (1:1 ratio), and the other two pieces had one part with eighth notes and one part with quarter notes, employing a ratio of 2:1 or 1:2 (upper : lower part). The pianists sat next to each other at the same keyboard, and each part was performed with the pianists' right hand. ${ }^{2}$ The pieces were designed to require no fingers to pass over or under each other so that the fingertips could be seen by the motion trackers. There were no immediate note repetitions in any of the pieces.

\section{Design}

The two pianists in each pair were assigned musical roles as the leader (or "soloist," performing the upper part) or follower ("accompanist," performing the lower part). Those roles were reversed within a session, so that all participants performed in all conditions in a withinsubject design. The assignment of the upper part to the leader role was chosen because the primary melodic line is usually in the upper register of duet performances, and in order to keep the number of conditions manageable. Two additional independent variables were auditory feedback and note ratio between the two parts (1:1, $2: 1$, or $1: 2$ ). There were four auditory feedback conditions: full feedback (both pianists heard both parts), one-way feedback (the leader heard only him/herself while the follower heard both parts), self-feedback (both pianists performed simultaneously but heard only themselves playing; see Figure 1a), and a baseline condition, in which each pianist performed his/her own part alone. Thus, the within-subjects design yielded a 4 (auditory feedback) $\times 3$ (note ratio) $\times 2$ (role) design .

\section{Equipment}

An active motion capture system (NDI Optotrak Certus with two trackers) monitored the movements of the pianists at a frame rate of $200 \mathrm{~Hz}$. Markers were placed on each fingernail tip of the pianists' right hand, on the wrist (ulna), and on a head strap on the forehead, approximately one inch $(2.5 \mathrm{~cm})$ above each eye.

\footnotetext{
${ }^{2}$ An alternative configuration in which the left hand performed the lower part and the right the upper part would have caused insufficient space for the pianists at the same keyboard.
} 
a) Auditory Feedback

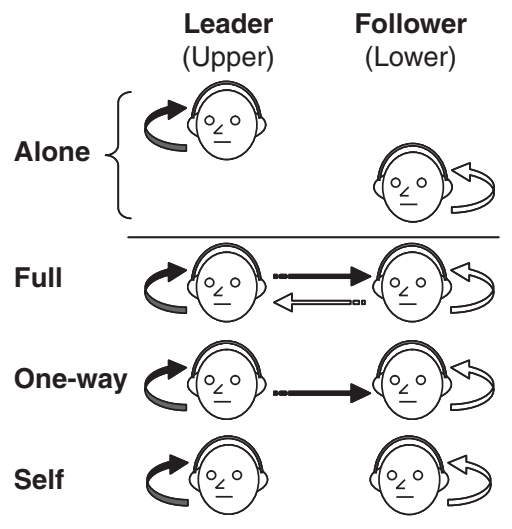

b) Note Ratio (Leader : Follower)
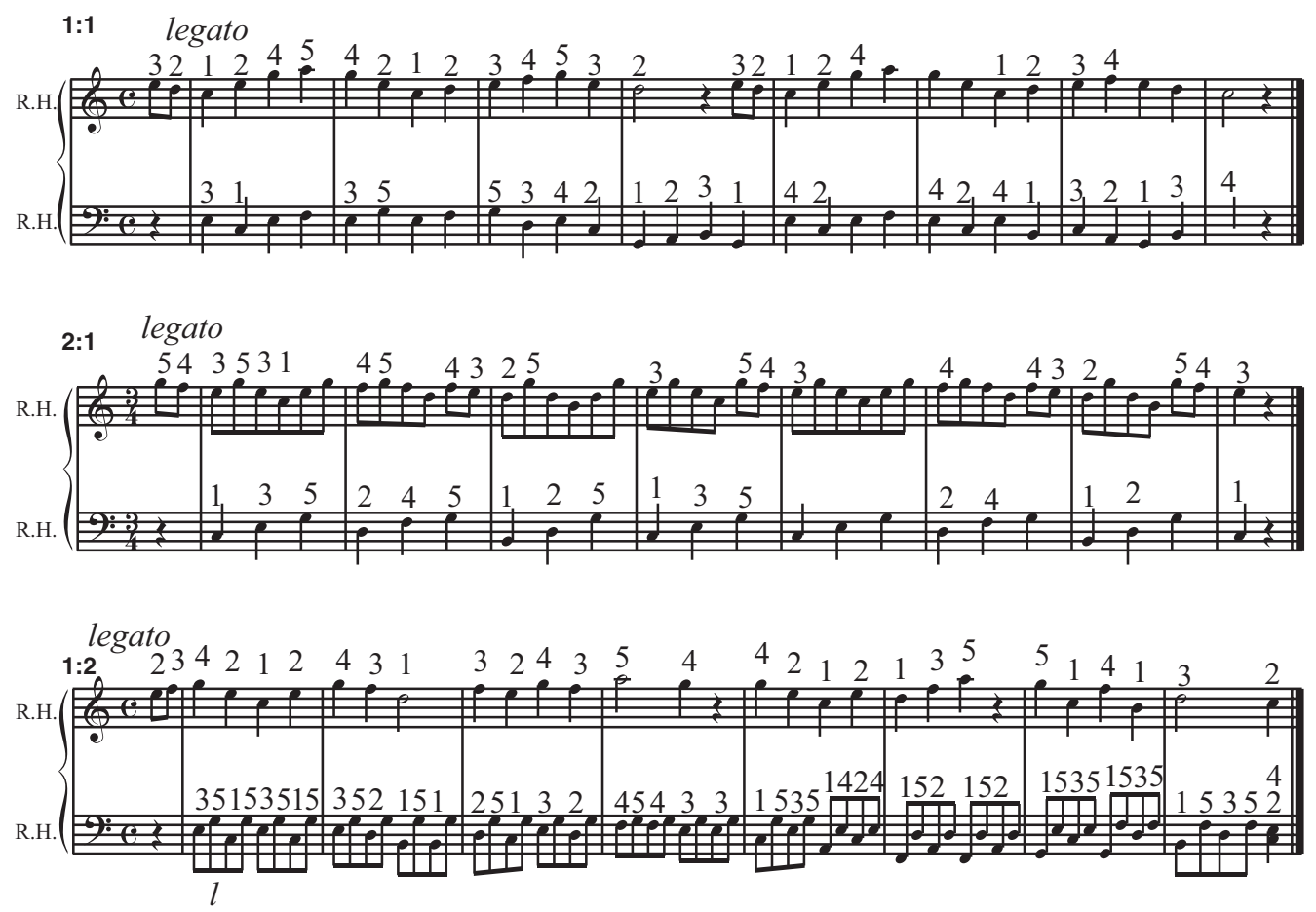

FIGURE 1. Schematic of the experimental conditions for auditory feedback (top) and note ratio (bottom).

Additional markers on the keyboard provided a reference frame. The MIDI output of the (silent) digital piano (Roland RD 700SX) was recorded to a computer that generated the auditory feedback manipulation using the linux-based software "ftap" (Finney, 2001). ${ }^{3}$ The piano sounds were produced by an Edirol Studio

\footnotetext{
${ }^{3}$ Ftap is reported to provide processing delays of about $1 \mathrm{~ms}$. The MIDI cables could introduce another $2 \mathrm{~ms}$ of delay if two keystrokes coincide exactly due to the serial nature of the transmission protocol.
}

Canvas SD-80 tone generator and heard over closed headphones (AKG K-271).

\section{Procedure}

The participants first completed a musical background questionnaire, were assigned a musical part that implied a role as leader or follower, and were equipped with the motion capture markers. They were then required to practice the parts, presented in music notation, both 
alone and together with full feedback. Each trial began with a metronome that sounded the quarter-note beats with $450 \mathrm{~ms}$ interonset intervals (133 beats per minute) for two bars and was then turned off. The participants were instructed to continue the tempo of the metronome. Each pianist first recorded two renditions of his/her part alone. Then, they recorded two trials together with full feedback, two with one-way feedback, and finally two with self-feedback. This order of the auditory feedback conditions was kept constant and was blocked within each of the three pieces (note ratio manipulation). The presentation order of pieces followed a Latin-square design. After all experimental conditions were completed, the two pianists swapped roles and sitting positions in front of the keyboard and repeated the same procedure. The entire experiment lasted about 90 minutes.

\section{Data Analysis}

The asynchronies of MIDI note onsets between the two parts were analyzed at the quarter-note level. Signed asynchronies were computed by subtracting the onset times of the upper part (leader) from the lower part (follower); thus, a positive asynchrony denotes that the pianist performing the upper part (the leader) was early and a negative asynchrony denotes that the follower was early. The asynchronies were analyzed both in milliseconds and in percentages of the preceding interonset interval of the leader's performance. As both ways of looking at the data delivered basically identical results (including all ANOVAs), we only report asynchronies in milliseconds.

The motion data of the finger and head movements were analyzed using Functional Data Analysis techniques (Ramsay \& Silverman, 2005). The coordinate system of the capture volume was aligned relative to the keyboard markers such that the $\mathrm{z}$ Dimension described the height above keyboard and the $y$ Dimension described the anterior-posterior movement of the player (the $\mathrm{x}$ Dimension described leftright movement). Kinematic landmarks (acceleration peaks ${ }^{4}$ associated with impacts on key surface and keybed) were identified in the finger trajectories before smoothing. One such landmark was the key-bottom contact $(\mathrm{KB})$ that was identified for every keystroke; in addition, a finger-key landmark (FK) was identified in those trajectories that had an acceleration peak larger than $10 \mathrm{~m} / \mathrm{s}^{2}$ in a time window of $30-150 \mathrm{~ms}$

\footnotetext{
${ }^{4}$ As the motion of the finger pressing a piano key is directed downwards, the acceleration of the finger during a keystroke is negative. Thus, the abrupt reductions in finger velocity (decelerations) due to impacts on the key surface or keybed are characterized by positive acceleration peaks.
}



b)

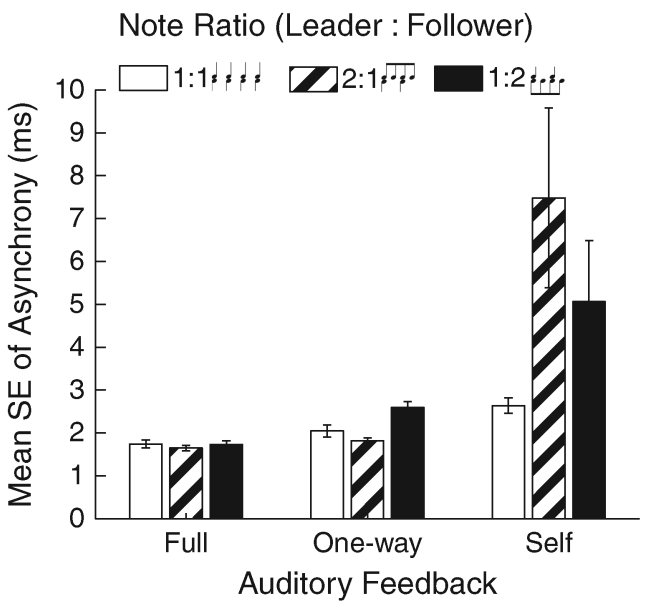

FIGURE 2. Mean asynchronies (a) and mean standard error of the asynchronies (b) in milliseconds, shown by note ratio and auditory feedback. Error bars denote the standard error of the means.

before a note onset (Goebl \& Palmer, 2008). Finally, the maximum height of the finger trajectory (called finger height, $\mathrm{FH}$ ) was extracted in a time window of up to two quarter notes before every keystroke. The head motion was derived from the averaged trajectories of the two head markers.

\section{Results \\ Timing}

The mean signed asynchronies are shown in Figure 2a by auditory feedback condition and note ratio. A twoway repeated measures analysis of variance (ANOVA) revealed a significant main effect of note ratio, $F(2,30)=$ $20.19, p_{a d j}<.001,{ }^{5}$ and a significant interaction of note

\footnotetext{
${ }^{5}$ The $p_{\text {adj }}$ values reflect the adjustments according to the Greenhouse-Geisser correction for sphericity violations.
} 
ratio with feedback, $F(4,60)=16.12, p_{a d j}<.001$. The main effect of feedback approached significance, $F(2$, $30)=3.62, p_{a d j}=.06$. The main effect of note ratio showed that the 1:1 condition was closest to synchrony, while the main effect of feedback tended to be largest in the one-way condition $(M=-12.7 \mathrm{~ms})$, indicating that the follower preceded the leader when the leader did not hear the follower's sound. However, the interaction revealed a more complex picture: Although note ratio did not affect asynchronies under full auditory feedback, posthoc analyses indicated it reached significance in both one-way, $F(2,30)=19.75, p_{a d j}<.001$, and self-feedback, $F(2,30)=18.30, p_{a d j}<.001$, conditions. In the one-way condition (in which the provided feedback enforced the role instructions), the follower was best synchronized (least asynchronous) when playing fewer notes (2:1 ratio) and worst synchronized when playing more notes (1:2 ratio). In the absence of auditory feedback (the self condition), the performer who had eighthnotes to play clearly preceded the other performer.

Figure $2 \mathrm{~b}$ shows the mean standard errors of the asynchronies by condition, which provide an alternative measure of synchronicity. The same ANOVA on the standard errors of the asynchronies revealed a significant effect of feedback, $F(2,30)=15.24, p_{a d j}<.001$, and an interaction of note ratio $\times$ feedback approaching significance, $F(4,60)=2.91, p_{a d j}=.09$. The variability of the asynchronies increased as the auditory feedback was reduced. To evaluate the patterns within the individual feedback conditions, planned comparisons were conducted. The note ratio conditions differed significantly only within the one-way feedback condition, $F(2,30)=13.19, p_{a d j}<.001 ; 6$ the $2: 1$ note ratio condition had significantly lower standard errors than the 1:2 condition, suggesting that producing more tones while receiving less auditory feedback hurt synchronization.

In addition, we tested how the interonset interval (IOI) timing of the two performers was influenced by the independent variables. First we considered the coefficient of variation $(\mathrm{CV}=\mathrm{SD} /$ Mean per trial $)$ of IOIs on the quarter-note level for each performer. The mean IOI across all performances was $436 \mathrm{~ms}$, slightly faster than the prescribed tempo (450 ms). Higher CVs may reflect more error correction in a produced sequence (Vorberg \& Schulze, 2002). Higher CVs were found in the duet performances for the follower $(M=.044)$ than for the leader $(M=.035), F(1,15)=13.98, p<.01$, suggesting more adjustment of timing by the follower.

\footnotetext{
${ }^{6}$ In the self-feedback condition, the ANOVA did not reach significance due to the large standard errors, $F(2,30)=2.61, p_{a d j}=.113$.
}

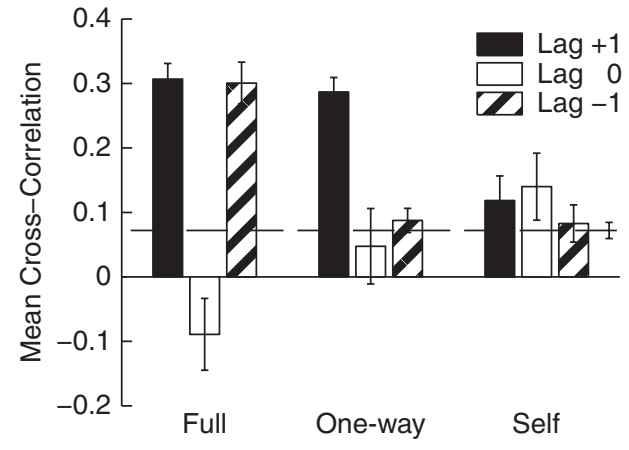

FIGURE 3. Mean cross-correlation coefficients of the interonset interval timing by auditory feedback and lag direction. A positive lag $(+1)$ indicates that the follower lags behind the leader; a negative lag $(-1)$ indicates a leader lagging behind the follower. The dashed line denotes the mean lag 0 cross-correlation of the alone performances. Error bars are standard errors of the means.

When the pianists played alone (upper alone $=.035$, lower alone $=.035$ ), their timing variability was low and similar to when they performed the upper part (leader) in the duet conditions.

To examine sequential influences of leader/follower roles on the timing of the two pianists, cross-correlations were computed between the individual quarter-note interonset intervals of the two performers. Cross-correlations at lag +1 and lag -1 examined tracking tendencies among the two pianists; to test for predictive behavior, lag- 0 cross-correlations served as comparison (Repp, 2002b). A lag +1 correlation was computed between the leader's IOI $\mathrm{I}_{1} \ldots(\mathrm{n}-1)$ and the follower's $\mathrm{IOI}_{2} \ldots \mathrm{n}$ ( $\mathrm{n}$ being the number of quarter-notes in a given melody), so that a positive coefficient would indicate the follower imitated the timing profile of the leader at a delay of one quarter note; positive values at lag -1 would indicate the inverse (that the leader tracked the follower's timing at a delay of one quarternote). Positive coefficients at lag 0 would indicate mutually predictive timing behavior. Figure 3 shows the mean cross-correlation coefficients for the three lag conditions $(+1,0,-1)$ by three auditory feedback conditions. The mean lag 0 cross-correlation value for the two "alone" performances (in which the pianists performed the parts at different times) is shown in Figure 3 by the dashed line, which serves as a baseline measure of timing similarity among the individual performers.

A two-way ANOVA on the cross-correlation coefficients, combined across the note ratio conditions, revealed significant main effects of feedback, $F(2,30)=$ 5.54, $p<.01$, lag direction, $F(2,30)=10.23, p<.001$, and a significant interaction, $F(4,60)=37.00, p<.001$. As shown in Figure 3, the values were largest during full feedback at both positive and negative lags, suggesting 
that shortened and lengthened IOIs tended to alternate (thus the two pianists tended to be in antiphase with each other). This pattern of correlations may indicate that pianists employ two-way tracking processes under full auditory feedback. The lag +1 and lag -1 conditions showed a significant difference only in the one-way condition (Tukey's HSD $=.10, p<.01$ ), suggesting that the pianists adopted the instructed musical roles (the follower followed the leader) only when enforced by one-way auditory feedback. In the absence of auditory feedback (self-feedback), no tracking occurred in any direction. The lag 0 correlations were an estimate of mutually predictive timing behavior. Although a separate ANOVA on the lag 0 coefficients revealed a significant main effect of auditory feedback, $F(2,30)=46.55, p<$ .001 , the values were not significantly larger than baseline during full and one-way auditory feedback and indicated equal amounts of prediction and tracking in the self-feedback condition. The correlation coefficients in the alone condition differed only from values in the full feedback and one-way $($ lag +1$)$ conditions (those conditions in which one or both pianists' timing was expected to be adjusted interactively).

\section{Finger Motion}

Next we examined the effects of auditory feedback and note ratio on finger motion in the height $(\mathrm{z})$ dimension above the keyboard plane. Three measures were computed and investigated from the landmarks of each finger trajectory toward a key: the percentage of keystrokes containing a finger-key (FK) landmark, the peak finger height above key surface prior to keystroke $(\mathrm{FH})$, and the finger movement duration of a keystroke (from peak finger height to key-bottom).

The FK landmark is indicative of the type of touch used for a particular keystroke. On average, $51.4 \%$ of all produced keystrokes contained a FK landmark (indicating that the key was played with a struck touch). The mean proportion of keystrokes containing FK landmarks are shown in Figure 4a, separately for note ratio and role. A three-way ANOVA on the percentage of FK keystrokes in each performance with feedback, note ratio, and role (leader/follower) as within-subject factors revealed a significant effect of note ratio, $F(2,30)=11.32$, $p<.001$, and a significant interaction of note ratio and role, $F(2,30)=98.94, p<.001$. The parts with more notes (essentially yielding a faster tempo) had considerably more landmarks (as expected from Goebl and Palmer's, 2008, previous results, showing effects of performance tempo on FK), whereas the 1:1 condition revealed more FK landmarks in the upper than in the lower part. The

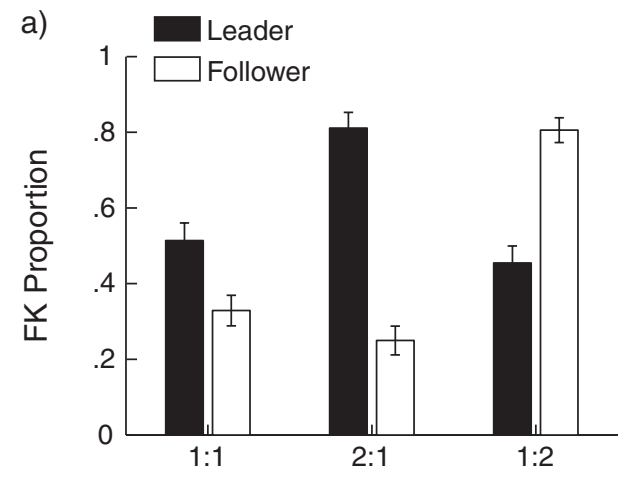

b)

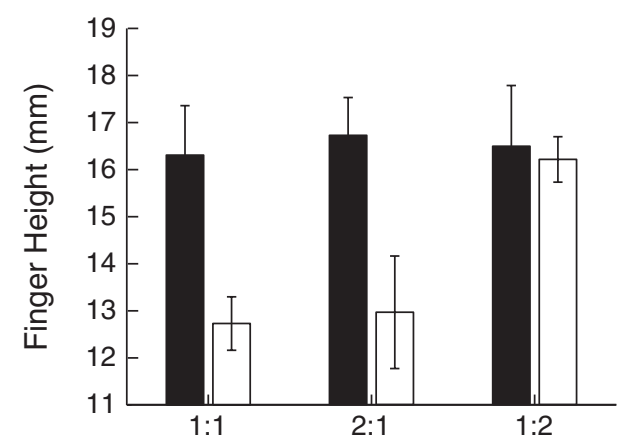

c)

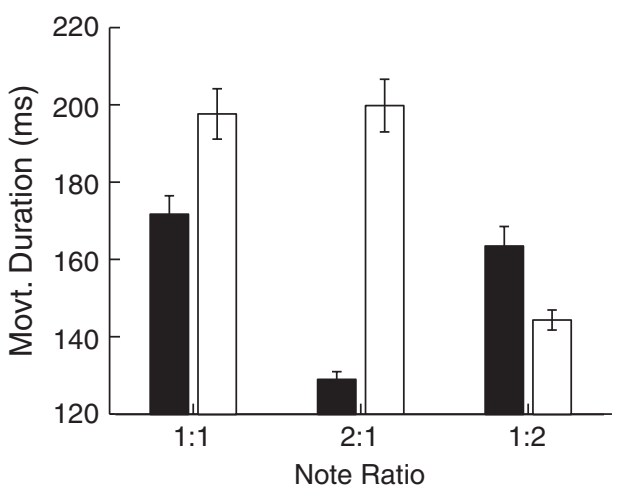

FIGURE 4. Main effects of auditory feedback (left column) and significant interactions of note ratio and musical role (right column) for the proportion of finger-key landmarks (FK proportion, top), peak finger height (middle), and movement duration (bottom). Error bars are standard errors of the means.

effect of auditory feedback was not significant, $F(2,30)=$ $2.84, p=.07$ (values shown in Table 1a).

The pianists' peak finger height prior to a keystroke $(\mathrm{FH})$ indicates how high the pianists raised the fingers above the key surface (in $\mathrm{mm}$ ) before actually playing a particular tone. Raising the fingers higher might be a way to communicate timing visually to the partner. A three-way ANOVA on peak finger heights revealed significant main effects of feedback, $F(2,30)=3.61, p<.05$, note ratio, $F(2,30)=3.86, p<.05$, and an interaction of 
TABLE 1. (a) Main effects of auditory feedback for the proportion of finger-key landmarks in the finger trajectories (FK proportion), the peak finger heights prior to a keystroke, and the movement duration. The numbers denote means and the standard errors in brackets. (b) Role $\times$ auditory feedback interaction for movement duration (ms).

(a)

\begin{tabular}{lrrr} 
& \multicolumn{3}{c}{ Auditory Feedback } \\
\cline { 2 - 4 } & \multicolumn{1}{c}{ FULL } & \multicolumn{1}{c}{ ONE-WAY } & \multicolumn{1}{c}{ SELF } \\
\hline FK Proportion & $.54(.04)$ & $.53(.04)$ & $.52(.04)$ \\
Finger Height (mm) & $15.15(.70)$ & $15.58(.71)$ & $14.99(.66)$ \\
Movement Duration (ms) & $162.71(4.31)$ & $168.58(4.29)$ & $171.69(4.50)$ \\
\cline { 2 - 4 }
\end{tabular}

(b) Movement Duration (ms)

\begin{tabular}{lllll}
\cline { 3 - 4 } Role & LEADER & $147.98(4.85)$ & $156.71(4.86)$ & $159.49(5.01)$ \\
& FOLLOWER & $177.44(6.50)$ & $180.44(6.68)$ & $183.89(7.09)$ \\
\hline
\end{tabular}

note ratio with role, $F(2,30)=6.24, p<.01$. None of the other effects or interactions reached significance. The main effect of feedback (Table 1a) showed highest peak amplitudes in the one-way feedback condition and smallest amplitudes in the self-feedback condition, suggesting that larger finger movements may have been used to communicate in the one-way condition than in the self condition (Tukey's HSD $=.57, p<.05$ ). The interaction of note ratio and role, shown in Figure $4 \mathrm{~b}$, suggests that the leader raised the fingers higher on average but not when the follower played eighth notes (1:2 note ratio).

The finger movement duration was defined as the time interval from the peak finger height to key-bottom (KB) in milliseconds, which may be considered a measure of directness (short values) or hesitancy (long values) of a keystroke. It is related to the intensity of the individual tones (as measured by MIDI velocities). A three-way ANOVA on movement duration yielded three significant main effects, feedback, $F(2,30)=$ $43.66, p<.001$, note ratio, $F(2,30)=17.15, p<.001$, and role, $F(1,15)=8.07, p<.05$, as well as a note ratio $\times$ role interaction, $F(2,30)=46.83, p<.001$ and a feedback $\times$ role interaction, $F(2,30)=3.38, p<.05$. No other interactions reached significance. Overall, the followers showed longer movement durations than the leaders. This main effect was modulated by note ratio: whichever part had more notes had shorter movement durations (Figure 4c). The main effect of auditory feedback (Table 1a) showed monotonically increasing keystroke durations as the feedback was reduced. This main effect may be indicative of more hesitations in the finger movements when auditory feedback was restricted. The interaction of feedback and role (Table 1b) shows that the difference in movement duration between leader and follower is largest under natural feedback condition, and smaller as auditory feedback is reduced.

Overall, the finger movements revealed stronger influences of how many notes had to be performed, and weaker effects of reduced auditory feedback, than did the timing data.

\section{Head Motion}

Finally, we tested whether head motion, as a measure of nonverbal communication, is influenced by auditory feedback or note ratio. We focused on the head motion in the y dimension (towards the keyboard, or anterior/posterior body sway), which was less constrained by the proximity of the other pianist than the other dimensions. Figure 5 shows the position and acceleration (second derivative) of the back-and-forth head movements of two performers playing the 1:1 note ratio under the three feedback conditions. This example represents a typical case in which the head movements became more synchronized as the auditory feedback was reduced (see below).

To test the head sway cohesion, the acceleration trajectories of the two pianists' head markers over the duration of the entire piece were subjected to cross-correlation analyses. Acceleration has been indicated as a relevant kinematic dimension in visual communication of performance gestures (Luck \& Sloboda, 2008). A cross-correlation profile of the acceleration trajectories was calculated up to a maximum lag of $+/-450 \mathrm{~ms}$ (corresponding to one metronome beat or one quarter 

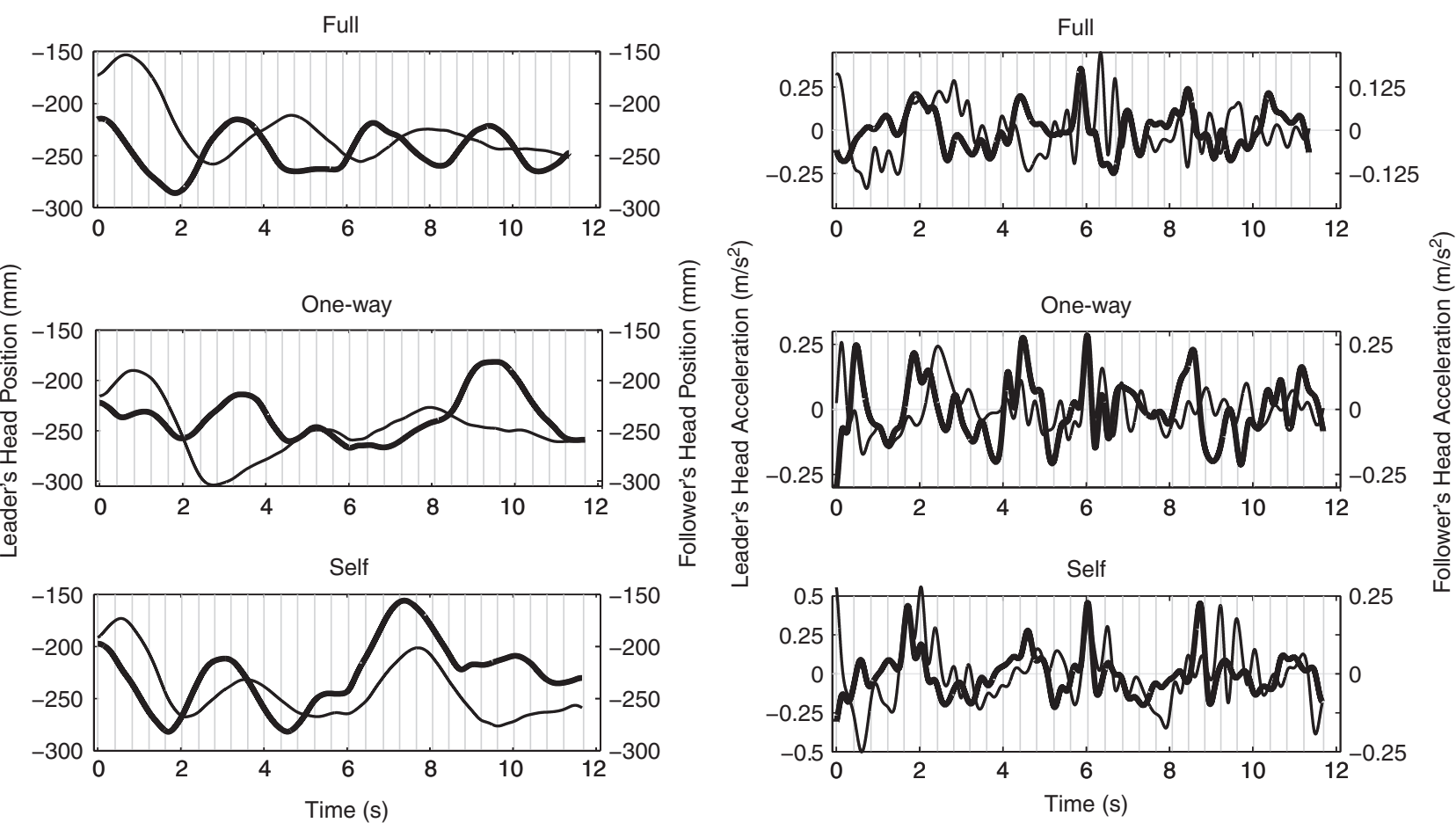

FIGURE 5. Head movements in the y dimension (anterior/posterior body sway) of one duet playing the 1:1 note ratio condition under full (top row), one-way (middle row), and self-feedback (bottom row). The left panels depict head position, the right panels depict acceleration. The leader's motion is depicted on the left y axes and with bold lines, the follower's on the right y axes and with thin lines. The vertical background lines indicate the quarternote onset times in the performances.

note). The maximum cross-correlation value across this interval on each trial was entered in a two-way ANOVA with the independent variables of auditory feedback (3) and note ratio (3). The ANOVA revealed a main effect of auditory feedback, $F(2,30)=6.10, p<.01$, and note ratio, $F(2,30)=4.97, p<.05$. Mean correlation coefficients increased monotonically as the auditory feedback was reduced (full $=.31$; one-way $=.34$; self $=.36$, all significantly greater than zero, $p$ 's $<.01$ ), suggesting as in Figure 5 that the body movements of the two players showed more agreement as auditory information was reduced. Pianists synchronized significantly more with their heads in the 2:1 note ratio condition $(M=.39)$ than in the other note ratio conditions $(1: 2 M=.32,1: 1$ $M=.30$ ). A similar ANOVA on the mean time lag at which the peak cross-correlations occurred revealed no significant effects of auditory feedback, note ratio, or interaction. Also shown in Figure 5 is the fact that the leader's head movements preceded those of the follower. The overall mean time lag was $60 \mathrm{~ms}$, indicating that the leader's head movements slightly preceded those of the follower, $t(15)=2.05, p=.059$ (two-tailed). Overall, the cross-correlations of head movements and timing showed inverse effects of reduced auditory feedback: As auditory feedback was reduced between performers, their timing synchronization decreased and their head movement synchronization increased.

\section{Discussion}

This study investigated the effects of auditory feedback, musical role, and note ratio on pianists' precision of synchronization in duet playing. Although a few studies have addressed ensemble performance, none have investigated the synchronization of note timing and of motion simultaneously among performing musicians. We investigated timing and synchronization aspects as well as finger kinematics and head motion in duet piano performance.

Auditory feedback was important for precise ensemble synchronization, even when participants sat next to each other and could see each other's movements. Overall, pianists' temporal synchronization levels were more accurate than those reported in other musical ensembles (Rasch, 1979) or in previous studies of piano duet playing (Shaffer, 1984); these differences might be due to different musical materials, instrumental timbres, ensemble sizes, and/or different methods of measurement. Pianists synchronized well when at least one performer could hear the other; in these 
conditions the mean asynchrony was below the perceptual threshold for detecting asynchronous onsets ( $30 \mathrm{~ms}$, Goebl \& Parncutt, 2002). In the absence of auditory feedback, the mean absolute asynchronies increased dramatically; this may be because they were not limited in this condition by feedback-related perceptual thresholds.

In addition to altered timing behavior, pianists' finger and head motions changed as auditory feedback was reduced. Pianists' finger heights above the keys increased in magnitude under reduced auditory feedback, potentially to communicate movement timing to the other pianist. The influence of removed auditory feedback on movements was most apparent in pianists' head movements, which became more synchronized with reduced feedback. The augmented visual information from these altered movements may serve to compensate to some extent for the lack of auditory feedback. However, head movement cues did not suffice in the absence of auditory information, as the synchronization measures clearly indicated. Additional measures of body sway synchronicity (such as those derived from torso or arm movements) may further address the potential causal relationship between restricted auditory feedback and bodily communication found in the current study.

Pianists were instructed to assume musical roles as leader and follower during their performances. Analyses of the timing variability suggested that playing with another performer affected the follower more than the leader; the follower's timing was less precise when playing with the leader than when playing alone. This supports an error correction interpretation of the timing variability: the follower adapted his or her timing more than did the leader. Furthermore, the sequential patterning of interonset timing indicated a directional bias in the tracking behavior between performers. When both pianists heard each other, they tracked each other's timing to the same extent, despite the instructed roles of leader and follower. When the follower heard both parts but the leader heard only his or her own part, the follower tracked the leader, but not vice versa. This suggests that auditory information is necessary for accurate cooperative timing of performance. Participants' comments on the instructed roles coincided with this finding; they said they would generally prefer musical cooperation over strict roles when playing together in an ensemble. These findings are also in line with evidence from tapping experiments that phase correction operates on a fairly automatic level (Repp, 2002a; Repp \& Keller, 2004). The performers' assigned musical roles also affected their motion: The leader's head movements tended to precede those of the follower, and more so when the two pianists synchronized their motion. The leader also tended to raise the fingers higher above the keyboard than the follower; the keystroke movements of the follower needed more time than those of the leader, indicating a potential role of this visual information as an alternative method of communication in the absence of auditory feedback.

Finally, the note ratio between the musical parts influenced ensemble synchronization: Whoever performed more notes tended to lead, particularly when auditory feedback was limited. This might be due to a tendency to play the smaller note values too short, consistent with recent findings that metrical subdivision slows the perceived beat by about $3-5 \%$ of the interbeat interval and thus speeds up the performed tempo (Repp, 2008). In the context of the present study, this metrical subdivision effect becomes apparent only when the phase correction mechanism of the pianist is inhibited by missing auditory feedback of the other player. Furthermore, the 2:1 note ratio condition generated less asynchrony during one-way feedback than did the 1:1 or $1: 2$ note conditions, indicating that pianists found it easier to synchronize fewer notes to more notes than the reverse. This is consistent with Repp's (2003) finding of a subdivision benefit in the variability of a 2:1 tapping condition relative to those of a 1:1 synchronization. However, subdividing a beat during production (1:2 note ratio) should also improve synchronization accuracy (Pfordresher \& Palmer, 2002; Wohlschläger \& Koch, 2000), which was not found in the present study. Translated into musical terms, it would be particularly challenging for an accompanist to fit the multiple notes of the piano part into the few melody notes of a typical solo part and predict upcoming events, especially when the soloist did not react to the accompanist.

Pianists' finger movements also were modulated by the note ratio between the musical parts. The percentage of keystrokes containing a finger-key (FK) landmark was higher for whoever performed the shortest durations. This can be explained by the rate of movement: faster rates entail larger FK proportions (Goebl \& Palmer, 2008). A rate effect also was found in the finger amplitudes: the fingers were raised higher above the keyboard when pianists had to play eighth notes (which are produced at twice the rate as the quarter notes), consistent with findings of Palmer and Dalla Bella (2004). Finally, finger movement durations of the follower were longer than those of the leader, but not when eighth notes were played. Overall, finger movements were more influenced by stimulus constraints than by auditory feedback or musical roles. 
In summary, synchronization behavior in ensemble performance shows evidence of complex crossperformer interactions with regard to both timing and motion. This study investigated three important variables-auditory feedback, musical role, and note ratio-that influenced the synchronization of note timing and finger and body motion. Although the findings of the present study may reflect the specifics of the experimental context including the compositional style and instrument, they provide a better understanding of interpersonal communication in realistic music performance. The perception of other performers' motion by the ensemble members altered their subsequent timing; likewise, the auditory perception of others' timing altered their subsequent motion. These findings suggest that ensemble performance offers an excellent testing ground for theories of human sensorimotor synchronization.

\section{Author Note}

This research was supported in part by a Schrödinger Fellowship from the Austrian Science Foundation to the first author and by NSERC Grant 298173 and a Canada Research Chair to the second author. We thank Rachel Brown, Bruno Repp, Martina Rieger, and two anonymous reviewers for valuable comments on earlier drafts of this manuscript.

Correspondence concerning this article should be addressed to Werner Goebl, Department of Computational Perception, Johannes Kepler University Linz, Altenberger Strasse 69, 4040 Linz, Austria; E-MAIL: werner.goebl@jku.at or Caroline Palmer, Department of Psychology, McGill University, 1205 Dr. Penfield Avenue, Montreal, QC H3A 1B1 Canada; E-MAIL: caroline.palmer@mcgill.ca

\section{References}

Altenmüller, E., Wiesendanger, M., \& Kesselring, J. (Eds.). (2006). Music, motor control, and the brain. Oxford, New York: Oxford University Press.

Aschersleben, G., \& Prinz, W. (1995). Synchronizing actions with events: The role of sensory information. Perception and Psychophysics, 57, 305-317.

Castellano, G., Mortillaro, M., Camurri, A., Volpe, G., \& Scherer, K. (2008). Automated analysis of body movement in emotionally expressive piano performances. Music Perception, 26, 103-120.

DAhL, S., \& FriberG, A. (2007). Visual perception of expressiveness in musicians' body movements. Music Perception, 24, 433-454.

DAVIDSON, J. W. (1993). Visual perception of performance manner in the movements of solo musicians. Psychology of Music, 21, 103-113.

Davidson, J. W., \& Good, J. M. M. (2002). Social and musical co-ordination between members of a string quartet: An exploratory study. Psychology of Music, 30, 186-201.

Delalande, F. (1988). La gestique de Gould [The gestures of Gould]. In G. Guertin (Ed.), Glenn Gould: Pluriel [Glenn Gould: Plural] (pp. 85-111). Verdun, QC, Canada: Louise Courteau.

FInNEY, S. A. (1997). Auditory feedback and musical keyboard performance. Music Perception, 15, 153-174.

Finney, S. A. (2001). FTAP: A Linux-based program for tapping and music experiments. Behavior Research Methods, Instruments and Computers, 33, 65-72.
Finney, S. A., \& Palmer, C. (2003). Auditory feedback and memory for music performance: Sound evidence for an encoding effect. Memory and Cognition, 31, 51-64.

Goebl, W., \& Palmer, C. (2008). Tactile feedback and timing accuracy in piano performance. Experimental Brain Research, 186, 471-479.

Goebl, W., \& Parncutt, R. (2002, August). The influence of relative intensity on the perception of onset asynchronies. Paper presented at the Seventh International Conference on Music Perception and Cognition, Sydney, Australia.

Goodman, E. (2002). Ensemble performance. In J. Rink (Ed.), Musical performance: A guide to understanding (pp. 153-167). Cambridge, UK: Cambridge University Press.

KeLler, P. E. (2008). Joint action in music performance. In F. Morganti, A. Carassa, \& G. Riva (Eds.), Enacting intersubjectivity: A cognitive and social perspective to the study of interactions (pp. 205-221). Amsterdam: IOS Press.

Large, E. W., \& Jones, M. R. (1999). The dynamics of attending: How people track time-varying events. Psychological Review, 106, 119-159.

Large, E. W., \& Kolen, J. F. (1994). Resonance and the perception of musical meter. Connection Science, 6, 177-208.

Loehr, J. D., \& Palmer, C. (2007). Cognitive and biomechanical influences in pianists' finger tapping. Experimental Brain Research, 178, 518-528.

Loehr, J. D., \& Palmer, C. (2009). Subdividing the beat: Auditory and motor contributions to synchronization. Music Perception, 26, 415-425. 
Luck, G., \& Sloboda, J. A. (2008). Exploring the spatiotemporal properties of simple conducting gestures using a synchronizing study. Music Perception, 25, 225-239.

Maduell, M., \& Wing, A. M. (2007). The dynamics of ensemble: The case for flamenco. Psychology of Music, 35, 591-627.

MATES, J. (1994). A model of synchronization of motor acts to a stimulus sequence. I. Timing and error corrections. Biological Cybernetics, 70, 463-473.

Moore, G. (1979). Am I too loud? Memoirs of an accompanist. London: Hamish Hamilton.

Murnighan, J. K., \& Conlon, D. E. (1991). The dynamics of intense work groups: A study of British string quartets. Administrative Science Quarterly, 36, 165-186.

Palmer, C. (1997). Music performance. Annual Review of Psychology, 48, 115-138.

Palmer, C., \& Dalla Bella, S. (2004). Movement amplitude and tempo change in piano performance. Journal of the Acoustical Society of America, 115, 2590.

Pfordresher, P. Q., \& Palmer, C. (2002). Effects of delayed auditory feedback on timing of music performance. Psychological Research, 66, 71-79.

Ramsay, J. O., \& Silverman, B. W. (2005). Functional data analysis (2nd ed.). New York: Springer.

RASCH, R. A. (1979). Synchronization in performed ensemble music. Acustica, 43, 121-131.

Repp, B. H. (2002a). Automaticity and voluntary control of phase correction following event onset shifts in sensorimotor synchronization. Journal of Experimental Psychology: Human Perception and Performance, 28, 410-430.

REPp, B. H. (2002b). The embodiment of musical structure: effects of musical context on sensorimotor synchronization with complex timing patterns. In W. Prinz \& B. Hommel (Eds.), Common mechanisms in perception and action: Attention and performance (Vol. XIX, pp. 245-265). Oxford, UK: Oxford University Press.
Repp, B. H. (2003). Rate limits in sensorimotor synchronization with auditory and visual sequences: The synchronization threshold and the benefits and costs of interval subdivision. Journal of Motor Behavior, 35, 355-370.

Repp, B. H. (2005). Sensorimotor synchronization: A review of the tapping literature. Psychonomic Bulletin and Review, 12, 969-992.

Repp, B. H. (2008). Metrical subdivision results in subjective slowing of the beat. Music Perception, 26, 19-39.

Repp, B. H., \& Keller, P. E. (2004). Adaptation to tempo changes in sensorimotor synchronization: Effects of intention, attention, and awareness. The Quarterly Journal of Experimental Psychology A: Human Experimental Psychology, 57A, 499-521.

SHAFFer, L. H. (1984). Timing in solo and duet piano performances. Quarterly Journal of Experimental Psychology, 36A, 577595.

Vines, B., Krumhansl, C. L., Wanderley, M. M., \& LEVitin, D. J. (2006). Cross-modal interaction in the perception of musical performance. Cognition, 101, 80-113.

Vorberg, D., \& Schulze, H.-H. (2002). Linear phasecorrection in synchronization: Predictions, parameter estimation, and simulations. Journal of Mathematical Psychology, 46, 56-87.

Vorberg, D., \& Wing, A. (1996). Modeling variability and dependence in timing. In H. Heuer \& S. W. Keele (Eds.), Handbook of perception and action, Volume 2: Motor skills (pp. 181-262). San Diego: Academic Press.

Williamon, A., \& Davidson, J. W. (2002). Exploring coperformer communication. Musicae Scientiae, 6, 53-72.

WoHlschläger, A., \& Koch, R. (2000). Synchronisation error: An error in time perception. In P. Desain \& W. L. Windsor (Eds.), Rhythm perception and production (pp. 115128). Lisse: Swets \& Zeitlinger. 